\title{
THE OOGURI-VAFA METRIC, HOLOMORPHIC DISCS AND WALL-CROSSING
}

\author{
KWOKWAI CHAN
}

\begin{abstract}
Recently, Gaiotto, Moore and Neitzke [6] proposed a new construction of hyperkähler metrics. In particular, they gave a new construction of the Ooguri-Vafa metric, in which they came across certain formulas. We interpret those formulas as wall-crossing formulas that appear in the SYZ construction of instanton-corrected mirror manifolds. This reveals the close relation between the Ooguri-Vafa metric and nontrivial holomorphic discs with boundary in special Lagrangian torus fibers.
\end{abstract}

\section{Introduction}

This note grew out of an attempt to understand the relationship between the new construction of the Ooguri-Vafa metric by Gaiotto, Moore and Neitzke [6] and the wall-crossing formulas which appear in the instanton-corrected construction of mirror manifolds in examples investigated by Auroux [1], [2].

In their recent beautiful work [6], Gaiotto, Moore and Neitzke proposed a new construction of hyperkähler metrics on complex integrable systems. The simplest case of this construction reproduces the Ooguri-Vafa metric. ${ }^{1}$ This sheds new light on the understanding of the metric. In particular, one is naturally lead to certain formulas which resemble the wall-crossing formulas in Auroux's examples of the construction of instanton-corrected mirror manifolds [1], [2].

To connect these two constructions, we will study mirror symmetry for the OoguriVafa metric from the point of view of the SYZ Conjecture [17]. Recall that the naïve SYZ construction of mirror manifolds, namely, dualizing special Lagrangian torus fibrations (or so-called T-duality in physics), in general does not give the correct complex geometry of the mirror manifold. This is due to the presence of singular fibers and nontrivial holomorphic discs with boundary on special Lagrangian torus fibers. So we usually need to modify the gluing of complex charts of the mirror manifold by disc instanton corrections, according to certain wall-crossing formulas.

In this note, we show that the formulas which appear in Gaiotto-Moore-Neitzke's construction of the Ooguri-Vafa metric are equivalent to the wall crossing formulas which appear in Auroux's constructions. In particular, we will be able to see how the construction of the Ooguri-Vafa metric get contributions from corrections by disc instantons, i.e. nontrivial holomorphic discs with boundary in special Lagrangian torus fibers.

At this point, we shall mention that the idea to use wall-crossing formulas (or gluing formulas) to study the SYZ version of mirror symmetry was first suggested by

\footnotetext{
Received by the editors December 10, 2009.

${ }^{1}$ The original construction by Ooguri and Vafa [16] was done by applying the Gibbons-Hawking ansatz; see also Gross-Wilson [9].
} 
Kontsevich and Soibelman in [14] (see also [13]). These formulas were later generalized and applied by Gross and Siebert [8] in their construction of toric degenerations of Calabi-Yau manifolds and their mirrors from affine manifolds with singularities. The wall-crossing formulas which appear here and in Auroux's examples in [1], [2] are all special cases of the gluing formulas used by Kontsevich-Soibelman and Gross-Siebert.

We shall also emphasis that in all cases, the wall-crossing phenomena are of the same origin, although there are indeed two kinds of walls as distinguished by Kontsevich and Soibelman in [15]. The formulas which we are referring to here and those in Auroux's works correspond to crossing the wall of second kind (see p. 27 in [15]); while the formulas in Gaiotto-Moore-Neitzke [6] correspond to crossing the wall of first kind (see p.30 in [15]). ${ }^{2}$

In fact, wall-crossing formulas of first kind, which describe the jumping behavior of numerical Donaldson-Thomas invariants, play a key role in Gaiotto-Moore-Neitzke's construction of more general hyperkähler metrics. However, in the Ooguri-Vafa case, the numerical Donaldson-Thomas invariants do not jump (see Remark 3.2), so the wall-crossing formula of first kind is trivial. ${ }^{3}$ It is interesting to understand the relationship between wall-crossing formulas of first kind and the construction of instantoncorrected mirror manifolds. In particular, it is desirable to know what the numerical Donaldson-Thomas invariants are counting.

The rest of this note is organized as follows. In the next section, we briefly review Gaiotto-Moore-Neitzke's new construction of the Ooguri-Vafa metric. In Section 3, we proceed to explain why the wall-crossing formulas which appear in Section 2 can be interpreted as wall-crossing formulas which appear in Auroux's construction of instanton-corrected mirror manifolds.

\section{Review of a new construction of the Ooguri-Vafa metric by Gaiotto-Moore-Neitzke [6]}

This section is a very brief review of the construction of the Ooguri-Vafa metric using the method proposed by Gaiotto, Moore and Neitzke in their recent work [6]. For more details and construction of hyperkähler metrics on general complex integrable systems, we refer the reader to the original paper [6].

Let $B=\{b \in \mathbb{C}:|b|<r\}$ be the open disc centered at the origin with radius $r>0$, and let $B^{\prime}=B \backslash\{0\}$. Suppose that $\psi: M \rightarrow B$ is an elliptic fibration with a type $I_{1}$ singular fiber over $0 \in B$. Consider the local system $\Gamma=R^{1} \psi_{*} \mathbb{Z} \rightarrow B^{\prime}$, the generic fiber of which is given by $\Gamma_{b} \cong H^{1}\left(M_{b}, \mathbb{Z}\right)$, where $M_{b}=\psi^{-1}(b)$ is the fiber over $b \in B^{\prime}$. The monodromy of $\Gamma$ around $0 \in B$ is nontrivial and given by

$$
\begin{aligned}
\gamma_{e}(b) & \mapsto \gamma_{e}(b), \\
\gamma_{m}(b) & \mapsto \gamma_{m}(b)+\gamma_{e}(b),
\end{aligned}
$$

\footnotetext{
${ }^{2}$ In the physics literature, the wall of first kind is called the wall of marginal stability.

${ }^{3}$ In the language of Gross-Siebert [8], there are no scattering in the Ooguri-Vafa case, since there is only one singular point, and hence one wall, in the base affine manifold.
} 
where $\left\{\gamma_{e}(b), \gamma_{m}(b)\right\}$ is a symplectic basis of $H^{1}\left(M_{b}, \mathbb{Z}\right) .{ }^{4}$ This basis extends to local sections $\gamma_{e}, \gamma_{m}$ of $\Gamma$ over a small enough open subset $U \subset B^{\prime}$. Since $M_{U}=\Gamma_{U}^{\vee} \otimes_{\mathbb{Z}}$ $(\mathbb{R} / 2 \pi \mathbb{Z})$, the sections $\gamma_{e}, \gamma_{m}$ define local fiber coordinates

$$
\theta_{e}, \theta_{m}: M_{U} \rightarrow \mathbb{R} / 2 \pi \mathbb{Z},
$$

so that we have $\left[\left.d \theta_{e}\right|_{M_{b}}\right]=2 \pi \gamma_{e}(b),\left[\left.d \theta_{m}\right|_{M_{b}}\right]=2 \pi \gamma_{m}(b)$. Note that $\theta_{e}$ can be extended to a global function on $M$, while $\theta_{m}$ cannot because of the nontrivial monodromy $\theta_{m} \mapsto \theta_{m}+\theta_{e}$.

To construct a hyperkähler metric on $M$, we define a homomorphism $Z: \Gamma \rightarrow \mathbb{C}$ by setting

$$
\begin{aligned}
Z\left(\gamma_{e}(b)\right) & =b, \\
Z\left(\gamma_{m}(b)\right) & =\frac{1}{2 \pi i}\left(b \log \frac{b}{r}-b\right) .
\end{aligned}
$$

$Z$ is called the central charge in the physics literature. The functions $Z_{e}:=Z\left(\gamma_{e}(b)\right)$, $Z_{m}:=Z\left(\gamma_{m}(b)\right)$ are defined in this way so that they are compatible with the monodromy of $\theta_{e}, \theta_{m}$ respectively. Then, we can define two families of $\mathbb{C}^{*}$-valued functions $\chi_{e}^{\mathrm{sf}}(\zeta), \chi_{m}^{\mathrm{sf}}(\zeta)$ locally on $M$ :

$$
\begin{aligned}
\chi_{e}^{\mathrm{sf}}(\zeta) & =\exp \left[\frac{\pi}{\epsilon}\left(\zeta^{-1} Z_{e}+\zeta \bar{Z}_{e}\right)+i \theta_{e}\right], \\
\chi_{m}^{\mathrm{sf}}(\zeta) & =\exp \left[\frac{\pi}{\epsilon}\left(\zeta^{-1} Z_{m}+\zeta \bar{Z}_{m}\right)+i \theta_{m}\right],
\end{aligned}
$$

parameterized by $\zeta \in \mathbb{C}^{*}$. Here, $\epsilon>0$ is a constant. The functions $\chi_{e}^{\text {sf }}(\zeta), \chi_{m}^{\text {sf }}(\zeta)$ give the so-called semi-flat local coordinates on $M$. Notice that the coordinate $\chi_{e}^{\text {sf }}(\zeta)$ extends to a global function on $M$, while $\chi_{m}^{\text {sf }}(\zeta)$ has nontrivial monodromy around $0 \in B$ given by $\chi_{m}^{\mathrm{sf}}(\zeta) \mapsto \chi_{e}^{\mathrm{sf}}(\zeta) \chi_{m}^{\mathrm{sf}}(\zeta)$.

Now, consider the two-forms

$$
\Omega^{\mathrm{sf}}(\zeta)=\frac{d \chi_{e}^{\mathrm{sf}}(\zeta)}{\chi_{e}^{\mathrm{sf}}(\zeta)} \wedge \frac{d \chi_{m}^{\mathrm{sf}}(\zeta)}{\chi_{m}^{\mathrm{sf}}(\zeta)}, \zeta \in \mathbb{C}^{*}
$$

on $M .^{5}$ In [6], it was checked that this family of two-forms $\left\{\Omega^{\mathrm{sf}}(\zeta): \zeta \in \mathbb{C}^{*}\right\}$ satisfies all the hypotheses in the theorem of Hitchin et al [12] (see also [10]) and concluded that $M \times \mathbb{C} P^{1}$ equipped with $\left\{\Omega^{\mathrm{sf}}(\zeta): \zeta \in \mathbb{C}^{*}\right\}$ is the twistor space of a hyperkähler metric $g^{\text {sf }}$ on $M$ (so that $\Omega^{\text {sf }}(\zeta)$ is a holomorphic 2-form with respect to the complex structure parameterized by $\zeta$ ). However, since $\chi_{m}^{\mathrm{sf}}(\zeta)$ is not globally defined on $M$, this semi-flat metric $g^{\text {sf }}$ is singular at a point in the fiber over $0 \in B$.

To obtain a smooth hyperkähler metric on $M$, Gaiotto, Moore and Neitzke argued that we should modify the function $\chi_{m}^{\mathrm{sf}}(\zeta)$ by instanton corrections. (We need not correct the function $\chi_{e}^{\mathrm{sf}}(\zeta)$ and thus we shall set $\chi_{e}(\zeta)=\chi_{e}^{\mathrm{sf}}(\zeta)$.) They did so by solving a Riemann-Hilbert problem which is described as follows. Consider the following

\footnotetext{
"The subscripts "e" and " $\mathrm{m}$ " stand for "electric" and "magnetic" respectively, and $\Gamma$ is called the charge lattice in [6].

${ }^{5}$ The definitions of the holomorphic two-forms $\Omega^{\mathrm{sf}}(\zeta)$ and $\Omega(\zeta)$ here differ from those in [6] by multiplication by the constant $-\epsilon / 4 \pi^{2}$.
} 
rays in the $\zeta$-plane.

$$
\begin{aligned}
& l_{+}=\left\{\zeta \in \mathbb{C}^{*}: b / \zeta \in \mathbb{R}_{<0}\right\}, \\
& l_{-}=\left\{\zeta \in \mathbb{C}^{*}: b / \zeta \in \mathbb{R}_{>0}\right\} .
\end{aligned}
$$

These are called the BPS rays corresponding to the central charge $Z_{e}$. The RiemannHilbert problem then asks for a family of holomorphic functions $\left\{\chi_{m}(\zeta): \zeta \in \mathbb{C}^{*}\right\}$ on $M$, which are piecewise holomorphic in $\zeta \in \mathbb{C}^{*}$, such that the following two conditions are satisfied. ${ }^{6}$

(a) $\chi_{m}(\zeta)$ is discontinuous across the BPS rays $l_{ \pm}$in the following way: Let $\left(\chi_{m}(\zeta)\right)_{l_{+}}^{+},\left(\chi_{m}(\zeta)\right)_{l_{+}}^{-}$be the limit of $\chi_{m}(\zeta)$ as $\zeta$ approaches $l_{+}$in the clockwise and counter-clockwise direction respectively, and similarly, $\left(\chi_{m}(\zeta)\right)_{l_{-}}^{+}$, $\left(\chi_{m}(\zeta)\right)_{l_{-}}^{-}$be the limit of $\chi_{m}(\zeta)$ as $\zeta$ approaches $l_{-}$in the clockwise and counter-clockwise direction respectively. Then we require that

$$
\begin{aligned}
\left(\chi_{m}(\zeta)\right)_{l_{+}}^{-} & =\left(\chi_{m}(\zeta)\right)_{l_{+}}^{+}\left(1+\chi_{e}(\zeta)\right), \\
\left(\chi_{m}(\zeta)\right)_{l_{-}}^{+} & =\left(\chi_{m}(\zeta)\right)_{l_{-}}^{-}\left(1+\chi_{e}^{-1}(\zeta)\right) .
\end{aligned}
$$

(b) Let

$$
\Upsilon(\zeta)=\chi_{m}(\zeta) \exp \left[-\frac{\pi}{\epsilon}\left(\zeta^{-1} Z_{m}+\zeta \bar{Z}_{m}\right)\right] .
$$

Then we require that the limit of $\Upsilon(\zeta)$ as $\zeta \rightarrow 0$ and $\zeta \rightarrow \infty$ exists, and the limits are related by

$$
\lim _{\zeta \rightarrow 0} \Upsilon(\zeta)=\overline{\lim _{\zeta \rightarrow \infty} \Upsilon(\zeta)}
$$

It is ingenious that Gaiotto, Moore and Neitzke were able to write down the following beautiful and explicit formula for $\chi_{m}(\zeta)$ in $[6] .^{7}$

$$
\begin{aligned}
\chi_{m}(\zeta)=\chi_{m}^{\mathrm{sf}}(\zeta) \exp \frac{i}{4 \pi}\left[\int_{l_{+}} \log \left(1+\chi_{e}\left(\zeta^{\prime}\right)\right) \frac{\zeta^{\prime}+\zeta}{\zeta^{\prime}-\zeta} \frac{d \zeta^{\prime}}{\zeta^{\prime}}\right. \\
\left.\quad-\int_{l_{-}} \log \left(1+\chi_{e}\left(\zeta^{\prime}\right)^{-1}\right) \frac{\zeta^{\prime}+\zeta}{\zeta^{\prime}-\zeta} \frac{d \zeta^{\prime}}{\zeta^{\prime}}\right]
\end{aligned}
$$

Now, the family of two forms

$$
\Omega(\zeta)=\frac{d \chi_{e}(\zeta)}{\chi_{e}(\zeta)} \wedge \frac{d \chi_{m}(\zeta)}{\chi_{m}(\zeta)}
$$

on $M$ again satisfies the hypotheses the theorem of Hitchin et al, and hence defines a smooth hyperkähler metric $g$ on $M$ (which can be determined explicitly from the family of two-forms $\left.\Omega(\zeta), \zeta \in \mathbb{C}^{*}\right)$. Furthermore, Gaiotto, Moore and Neitzke verified

\footnotetext{
${ }^{6}$ See Section 4.4 in [6] for details; due to the choice of the monodromy, our formulas $(2.1),(2.2)$ differ from the formulas $(4.52 \mathrm{a}),(4.52 \mathrm{~b})$ on p.16 of [6] by a sign.

${ }^{7}$ The generalization of this formula, which is an integral equation satisfied by the functions $\chi_{\gamma}(\zeta)$, turns out to be the key in the general construction of hyperkähler metrics on general complex integrable systems. In particular, one can obtain successive approximations of the desired hyperkähler metric by iteratively solving the integral equation.
} 
that this is nothing but the Ooguri-Vafa metric constructed by the Gibbons-Hawking ansatz [16] (see also Gross-Wilson [9]).

\section{Holomorphic discs, wall-crossing and SYZ mirror symmetry}

In this section, we study mirror symmetry for the Ooguri-Vafa metric from the viewpoint of the SYZ Conjecture [17] and interpret the formulas (2.1), (2.2), which describe the discontinuity of the function $\chi_{m}(\zeta)$ across the BPS rays $l_{ \pm}$, as wallcrossing formulas which appear in the SYZ construction of the instanton-corrected mirror manifold, following the approach of Auroux (see Section 5 in [1] and Section 3 in [2]). These wall-crossing phenomena are special cases of those studied first by Kontsevich and Soibelman in [14], which also played a crucial role in the foundational work of Gross and Siebert [8].

To begin with, recall that we have a family of two-forms $\left\{\Omega(\zeta): \zeta \in \mathbb{C}^{*}\right\}$ on $M$. For each $\zeta \in \mathbb{C}^{*}, \Omega(\zeta)$ is holomorphic with respect to a complex structure $J(\zeta)$, and there is a corresponding Kähler form $\omega(\zeta)$. We want to write down a formula for $\omega(\zeta)$. To do this, recall that, in the Gibbons-Hawking ansatz, the hyperkähler metric $g$ on $M$ is determined by a triplet of symplectic forms

$$
\begin{aligned}
& \omega_{1}=d b_{1} \wedge \alpha+V d b_{2} \wedge d b_{3}, \\
& \omega_{2}=d b_{2} \wedge \alpha+V d b_{3} \wedge d b_{1}, \\
& \omega_{3}=d b_{3} \wedge \alpha+V d b_{1} \wedge d b_{2},
\end{aligned}
$$

where $b=b_{1}+i b_{2} \in B, b_{3}=\frac{\epsilon \theta_{e}}{2 \pi} \in \mathbb{R} / \epsilon \mathbb{Z}, V=V\left(b_{1}, b_{2}, b_{3}\right)$ is a positive harmonic function on $(B \times \mathbb{R} \backslash\{0\} \times \epsilon \mathbb{Z}) / \epsilon \mathbb{Z}$, and $\alpha$ is a connection one-form on $M$ (which can be realized as a partial compactification of a circle bundle over $(B \times \mathbb{R} \backslash\{0\} \times \epsilon \mathbb{Z}) / \epsilon \mathbb{Z})$ of the form $\frac{d \theta_{m}}{2 \pi}+A\left(b_{1}, b_{2}, b_{3}\right)$ which satisfies $d \alpha=d A=\star d V$. There are explicit formulas for $V$ and $\alpha$, see Remark 3.3. The symplectic form $\omega(\zeta)$, which is Kähler with respect to $J(\zeta)$, is then given by

$$
\omega(\zeta)=\frac{4 \pi^{2}}{\epsilon}\left[\frac{i\left(\bar{\zeta} \omega_{+}-\zeta \omega_{-}\right)+\left(1-|\zeta|^{2}\right) \omega_{3}}{1+|\zeta|^{2}}\right],
$$

where $\omega_{ \pm}=\omega_{1} \pm i \omega_{2}$. We also have

$$
\Omega(\zeta)=-\frac{4 \pi^{2}}{\epsilon}\left[\frac{1}{2 i}\left(\zeta^{-1} \omega_{+}+\zeta \omega_{-}\right)+\omega_{3}\right] .
$$

Now, we shall fix $\zeta \in \mathbb{C}^{*}$ and denote by $M(\zeta)$ the manifold $M$ equipped with the Kähler form $\omega(\zeta)$ and the holomorphic two-form $\Omega(\zeta)$. We want to study the SYZ mirror symmetry for $M(\zeta)$. The first step is to construct a special Lagrangian torus fibration. Consider the $S^{1}$-action on $M$ given by rotating the angle coordinate $\theta_{m}$ :

$$
e^{i t} \cdot\left(b_{1}, b_{2}, \theta_{e}, \theta_{m}\right)=\left(b_{1}, b_{2}, \theta_{e}, \theta_{m}+t\right) .
$$

Lemma 3.1. This $S^{1}$-action is Hamiltonian with respect to $\omega(\zeta)$ when $|\zeta|=1$, and the moment map is then given by

$$
\mu_{S^{1}}=\frac{2 \pi}{\epsilon} \operatorname{Im}(\bar{\zeta} b): M \rightarrow \mathbb{R} .
$$


Proof. It is clear that the $S^{1}$-action preserves $\omega(\zeta)$. By a straightforward computation, we have

$$
\begin{aligned}
\omega(\zeta)=\frac{4 \pi^{2}}{\epsilon} d\left[\frac{-2 \operatorname{Im}(\bar{\zeta} b)+\left(1-|\zeta|^{2}\right) \frac{\epsilon \theta_{e}}{2 \pi}}{1+|\zeta|^{2}}\right] \wedge\left[\frac{d \theta_{m}}{2 \pi}+A\right] \\
+\frac{4 \pi^{2}}{\epsilon} V d \operatorname{Re}(\bar{\zeta} b) \wedge d\left[\frac{\frac{\epsilon \theta_{e}}{\pi}+|\zeta|^{-2}\left(1-|\zeta|^{2}\right) \operatorname{Im}(\bar{\zeta} b)}{1+|\zeta|^{2}}\right]
\end{aligned}
$$

Hence,

$$
\iota_{\frac{\partial}{\partial \theta_{m}}} \omega(\zeta)=\frac{2 \pi}{\epsilon} d\left[\frac{2 \operatorname{Im}(\bar{\zeta} b)-\left(1-|\zeta|^{2}\right) \frac{\epsilon \theta_{e}}{2 \pi}}{1+|\zeta|^{2}}\right],
$$

which is exact when $|\zeta|=1$, and the moment map is given by

$$
\mu_{S^{1}}=\frac{2 \pi}{\epsilon} \operatorname{Im}(\bar{\zeta} b)
$$

In view of the above lemma, we shall from now on fix a $\zeta$ such that $|\zeta|=1$.

Recall that we have a globally defined coordinate

$$
\chi_{e}(\zeta)=\exp \left[\frac{2 \pi}{\epsilon} \operatorname{Re}(\bar{\zeta} b)+i \theta_{e}\right]: M \rightarrow \mathbb{C}^{*},
$$

which is holomorphic with respect to the complex structure $J(\zeta)$.

Definition 3.1. For $(s, \lambda) \in \mathbb{R}^{2}$, define

$$
T_{s, \lambda}=\left\{\left(b_{1}, b_{2}, \theta_{e}, \theta_{m}\right) \in M: \log \left|\chi_{e}(\zeta)\right|=s, \mu_{S^{1}}=\lambda\right\} .
$$

For $(s, \lambda) \neq(0,0), T_{s, \lambda}$ is a torus embedded in $M$, and $T_{0,0}$ is nodal. Now, the reduced space $M_{r e d, \lambda}=\mu_{S^{1}}^{-1}(\lambda) / S^{1}$ is topologically an annulus, and from formula (3.1), we can see that the reduced holomorphic volume form is given by

$$
\Omega(\zeta)_{r e d, \lambda}=\iota_{\frac{\partial}{\partial \theta_{m}}} \Omega(\zeta)=-i d \log \chi_{e}(\zeta) .
$$

Thus, by Theorem 1.2 in Gross [7], we have the following result (see also Proposition 5.2 in Auroux [1]).

Proposition 3.1. Each $T_{s, \lambda}$ is special Lagrangian in $M$ with respect to $\omega(\zeta), \Omega(\zeta)$. Hence, the map $\Psi: M(\zeta) \rightarrow \mathbb{R}^{2}$ defined by

$$
\Psi=\left(\log \left|\chi_{e}(\zeta)\right|, \mu_{S^{1}}\right)
$$

gives a special Lagrangian torus fibration, with a single nodal fiber $T_{0,0}$.

In fact, we have

and thus

$$
\log \left|\chi_{e}(\zeta)\right|=\frac{2 \pi}{\epsilon} \operatorname{Re}(\bar{\zeta} b), \mu_{S^{1}}=\frac{2 \pi}{\epsilon} \operatorname{Im}(\bar{\zeta} b),
$$

$$
\Psi=\frac{2 \pi \bar{\zeta}}{\epsilon} \psi
$$


where $\psi: M \rightarrow B$ is the elliptic fibration that we start with. So the image of $\Psi$ is given by $\frac{2 \pi}{\epsilon} B=\left\{b \in \mathbb{C}:|b|<\frac{2 \pi r}{\epsilon}\right\}$. We will abuse notations and use $B$ to denote $\left\{b \in \mathbb{C}:|b|<\frac{2 \pi r}{\epsilon}\right\}$.

Now, as the base of a Lagrangian torus fibration, $B$ is a two-dimensional affine manifold with a unique singular point at $b=0 \in B$. This is called the focusfocus singularity in Hamiltonian mechanics (see for example Section 3 in Castaño Bernard-Matessi [3]). There are symplectic affine coordinates on $B$ defined as follows (see Hitchin [11] for details). First let $\left\{\gamma_{e}^{*}, \gamma_{m}^{*}\right\}$ be the basis of $H_{1}\left(T_{s, \lambda}, \mathbb{Z}\right)$ dual to $\left\{\gamma_{e}, \gamma_{m}\right\} \subset H^{1}\left(T_{s, \lambda}, \mathbb{Z}\right)$. For every tangent vector $\nu$ on $B$, lift it to a normal vector field (which we again denoted by $\nu$ ) on $T_{s, \lambda}$. Then the 1 -forms

$$
\omega_{e}(\zeta)(\nu)=\int_{\gamma_{e}^{*}} \iota_{\nu} \omega(\zeta), \omega_{m}(\zeta)(\nu)=\int_{\gamma_{m}^{*}} \iota_{\nu} \omega(\zeta)
$$

on $B$ are closed, and thus there are locally defined coordinates $\phi_{e}(\zeta), \phi_{m}(\zeta)$ on $B$ such that $d \phi_{e}(\zeta)=\omega_{e}(\zeta) / 2 \pi, d \phi_{m}(\zeta)=\omega_{m}(\zeta) / 2 \pi{ }^{8}$ These are called the symplectic affine coordinates on $B$ with respect to the basis $\left\{\gamma_{e}^{*}, \gamma_{m}^{*}\right\}$.

Proposition 3.2. The symplectic affine coordinates on $B$ with respect to the basis $\left\{\gamma_{e}^{*}, \gamma_{m}^{*}\right\}$ are explicitly given by

$$
\begin{aligned}
\phi_{m}(\zeta) & =-\frac{2 \pi}{\epsilon} \operatorname{Im}(\bar{\zeta} b) \\
\phi_{e}(\zeta) & =-\frac{1}{\epsilon} \operatorname{Re}\left[\bar{\zeta}\left(b \log \frac{b}{r}-b\right)\right] .
\end{aligned}
$$

Proof. Since $|\zeta|=1$, we have

$$
\omega(\zeta)=-\frac{4 \pi^{2}}{\epsilon} \operatorname{Im}\left(\bar{\zeta} \omega_{+}\right)=-\frac{4 \pi^{2}}{\epsilon} d \operatorname{Im}(\bar{\zeta} b) \wedge\left(\frac{d \theta_{m}}{2 \pi}+A\right)+2 \pi V d \operatorname{Re}(\bar{\zeta} b) \wedge d \theta_{e} .
$$

As $V=V\left(b_{1}, b_{2}, b_{3}\right)$ and $A=A\left(b_{1}, b_{2}, b_{3}\right)$ are independent of $\theta_{m}$ (recall that $b_{3}=$ $\left.\epsilon \theta_{e} / 2 \pi\right)$, it is easy to see that

$$
d \phi_{m}(\zeta)=\frac{\omega_{m}(\zeta)}{2 \pi}=-\frac{2 \pi}{\epsilon} d \operatorname{Im}(\bar{\zeta} b)
$$

where we use $\int_{\gamma_{m}^{*}} d \theta_{m}=\int_{\gamma_{e}^{*}} d \theta_{e}=2 \pi$. Hence, we can take $\phi_{m}(\zeta)=-\frac{2 \pi}{\epsilon} \operatorname{Im}(\bar{\zeta} b)$.

On the other hand, as will be seen in Remark 3.3, we can decompose $V$ and $A$ into sums of semi-flat and instanton parts, i.e. $V=V^{\mathrm{sf}}+V^{\text {inst }}, A=A^{\mathrm{sf}}+A^{\text {inst }}$. And observe that both $V^{\text {inst }}$ and $A^{\text {inst }}$ are periodic in $\theta_{e}$ and have no constant terms in their Fourier series expansions, so we have

$$
\int_{\gamma_{e}^{*}} \iota_{\nu} \omega(\zeta)=\int_{\gamma_{e}^{*}} \iota_{\nu}\left(-\frac{4 \pi^{2}}{\epsilon} d \operatorname{Im}(\bar{\zeta} b) \wedge A^{\mathrm{sf}}+2 \pi V^{\mathrm{sf}} d \operatorname{Re}(\bar{\zeta} b) \wedge d \theta_{e}\right) .
$$

\footnotetext{
${ }^{8}$ We normalize the coordinates $\phi_{e}, \phi_{m}$ so that the formulas appear later will be simpler.
} 
Now, by the explicit formulas for $V^{\text {sf }}$ and $A^{\text {sf }}$ in Remark 3.3, we compute

$$
\begin{aligned}
& -\frac{4 \pi^{2}}{\epsilon} d \operatorname{Im}(\bar{\zeta} b) \wedge A^{\mathrm{sf}}+2 \pi V^{\mathrm{sf}} d \operatorname{Re}(\bar{\zeta} b) \wedge d \theta_{e} \\
= & \frac{-i}{2 \epsilon}\left(\log \frac{b}{r}-\log \frac{\bar{b}}{r}\right) d \operatorname{Im}(\bar{\zeta} b) \wedge d \theta_{e}-\frac{1}{2 \epsilon}\left(\log \frac{b}{r}+\log \frac{\bar{b}}{r}\right) d \operatorname{Re}(\bar{\zeta} b) \wedge d \theta_{e} \\
= & -\frac{1}{\epsilon} d \operatorname{Re}\left[\bar{\zeta}\left(b \log \frac{b}{r}-b\right)\right] \wedge d \theta_{e} .
\end{aligned}
$$

Hence,

$$
d \phi_{e}(\zeta)=\frac{\omega_{e}(\zeta)}{2 \pi}=-\frac{1}{\epsilon} d \operatorname{Re}\left[\bar{\zeta}\left(b \log \frac{b}{r}-b\right)\right],
$$

and we can take $\phi_{e}(\zeta)=-\frac{1}{\epsilon} \operatorname{Re}\left[\bar{\zeta}\left(b \log \frac{b}{r}-b\right)\right]$.

\section{Remark 3.1.}

(1) Notice that the symplectic affine coordinates are of the form stated by Castaño Bernard-Matessi on p.511 in [3], as expected.

(2) In the same way, one can show that the complex affine coordinates (which correspond to periods of the form $\operatorname{Im}(\Omega(\zeta)))$, with respect to the basis $\left\{-\gamma_{e}^{*}, \gamma_{m}^{*}\right\}$, are given by

$$
\frac{2 \pi}{\epsilon} \operatorname{Re}(\bar{\zeta} b)=\log \left|\chi_{e}(\zeta)\right|, \frac{1}{\epsilon} \operatorname{Im}\left[\bar{\zeta}\left(b \log \frac{b}{r}-b\right)\right]=\log \left|\chi_{m}^{s f}(\zeta)\right| .
$$

(3) The central charge $Z: \Gamma \rightarrow \mathbb{C}$ satisfies the following relations:

$$
\int_{\gamma_{m}^{*}} \omega_{+}=\frac{1}{2 \pi} d Z_{e}, \int_{\gamma_{e}^{*}} \omega_{+}=-\frac{1}{2 \pi} d Z_{m} .
$$

If we define $\check{Z}: \Gamma^{\vee} \rightarrow \mathbb{C}$ by setting $Z\left(\gamma_{m}^{*}\right)=Z_{e}$ and $Z\left(\gamma_{e}^{*}\right)=-Z_{m}$, then $\check{Z}$ agrees with the definition of the central charge for a complex integrable system given by Kontsevich-Soibelman in Section 2.7 in [15].

Having constructed a special Lagrangian torus fibration $\Psi: M(\zeta) \rightarrow B$ and computed the symplectic affine coordinates on the base $B$, let us recall the construction of the mirror manifold $\check{M}(\zeta)$ (as a complex manifold) as suggested by the SYZ Conjecture [17]. First of all, consider the moduli space of pairs $\left(T_{s, \lambda}, \nabla\right)$, where $T_{s, \lambda}$ is a nonsingular special Lagrangian torus fiber and $\nabla$ is a flat $U(1)$-connection on the trivial complex line bundle over $T_{s, \lambda}$. The mirror manifold $\check{M}(\zeta)$ should be, at least topologically, a partial compactification of this moduli space. More precisely, $\check{M}(\zeta)$ should contain the quotient $T B^{\prime} / \Gamma$ of the tangent bundle of $B^{\prime}=B \backslash\{0\}$ by the lattice $\Gamma$ (while $M(\zeta)$ contains $T^{*} B^{\prime} / \Gamma^{\vee}$ ) as a dense open subset. And, given the symplectic affine coordinates $\phi_{m}(\zeta), \phi_{e}(\zeta)$ on $B^{\prime} \subset B$, the complex coordinates on $T B^{\prime} / \Gamma \subset \check{M}(\zeta)$ are naturally given by exponentiating the complexified coordinates, so we set

$$
w=\exp \left(\phi_{m}(\zeta)+i \check{\theta}_{m}\right), u^{\mathrm{sf}}=\exp \left(-\phi_{e}(\zeta)-i \check{\theta}_{e}\right) .
$$

These give local complex coordinates on the open dense subset $T B^{\prime} / \Gamma$ in the mirror manifold $\check{M}(\zeta)$. However, while the coordinate $w$ is globally defined on $\check{M}(\zeta)$, 
the other coordinate $u^{\text {sf }}$ does not extend to a global coordinate due to nontrivial monodromy around $b=0 \in B: u^{\mathrm{sf}} \mapsto u^{\mathrm{sf}} w .^{9}$

In fact, this is a general phenomenon: When the special Lagrangian torus fibration $M \rightarrow B$ admits singular fibers ( $T_{0,0}$ in our case), the local complex coordinates on the open dense subset $T B^{\prime} / \Gamma$ of the mirror $\check{M} \rightarrow B$ given by exponentiating the complexification of the symplectic affine coordinates on the smooth part $B^{\prime}$ of the base $B$ cannot be extended to the whole mirror manifold $\check{M}$ due to nontrivial monodromy around the singular locus $\Delta=B \backslash B^{\prime}$.

To obtain the correct complex coordinates on the mirror manifold, we must incorporate the information of the singular special Lagrangian fibers and nontrivial holomorphic discs with boundary on the smooth special Lagrangian torus fibers (disc instantons). More precisely, we need to modify the gluing of the local complex charts on the mirror manifold by disc instanton corrections according to certain wall-crossing formulas. This approach of constructing the corrected mirror manifolds was first suggested by Kontsevich and Soibelman in [14] in the two dimensional case (K3 surfaces). Later this was studied and generalized by Gross and Siebert [8] to higher dimensional cases. Explicit examples which indicate directly the relation of the gluing formulas to holomorphic discs instantons were first given by Auroux in [1], [2].

To carry out the construction of the instanton-corrected mirror manifold in our case, we shall first determine which special Lagrangian torus fibers bound nontrivial holomorphic discs. We have the following proposition (see also Lemma 5.4 in Auroux $[1])$.

Proposition 3.3. The special Lagrangian torus $T_{s, \lambda}$ bounds a nontrivial $J(\zeta)$ holomorphic disc $\varphi:\left(D^{2}, \partial D^{2}\right) \rightarrow\left(M(\zeta), T_{s, \lambda}\right)$ if and only if $s=0$.

Proof. Consider the map $f_{\zeta}=\chi_{e}(\zeta): M \rightarrow \mathbb{C}^{*}$. Note that the image of $f_{\zeta}$ is the annulus $\{a \in \mathbb{C}: \exp (-2 \pi r / \epsilon) \leq|a| \leq \exp (2 \pi r / \epsilon)\}$.

Now, suppose that $T_{s, \lambda}$ bounds a nontrivial holomorphic disc $\varphi:\left(D^{2}, \partial D^{2}\right) \rightarrow$ $\left(M(\zeta), T_{s, \lambda}\right)$. Then the composite map $f_{\zeta} \circ \varphi:\left(D^{2}, \partial D^{2}\right) \rightarrow\left(\mathbb{C}^{*},\left\{|a|=e^{s}\right\}\right)$ is a holomorphic map. By the maximum principle, $f_{\zeta} \circ \varphi$ must be a constant map. Hence, the image of the holomorphic disc is contained in some fiber of $f_{\zeta}$. However, for $a \neq 1$, the fiber $f_{\zeta}^{-1}(a)$ is biholomorphic to an annulus, and so cannot contain any nontrivial holomorphic disc. So we must have $e^{s}=1$ or $s=0$.

Conversely, observe that $f_{\zeta}^{-1}(1)$ is reducible and biholomorphic to the union of two discs. Thus, for $\lambda \neq 0, T_{0, \lambda}$ indeed bounds a nontrivial holomorphic disc which is contained entirely in the fiber $f_{\zeta}^{-1}(1)$.

We remark that, for $\lambda>0$, the special Lagrangian torus $T_{0, \lambda}$ boounds a nontrivial holomorphic disc with symplectic area $\lambda$. Denote by $\beta$ the relative homotopy class of

\footnotetext{
${ }^{9}$ Since the monodromy of $\theta_{e}, \theta_{m}$ around $b=0 \in B$ is given by the matrix $T=\left(\begin{array}{ll}1 & 1 \\ 0 & 1\end{array}\right)$, the monodromy of the dual coordinates $\check{\theta}_{e}, \check{\theta}_{m}$ should be given by the matrix $\left(T^{-1}\right)^{t}=\left(\begin{array}{cc}1 & 0 \\ -1 & 1\end{array}\right)$, i.e. $\check{\theta}_{e} \mapsto \check{\theta}_{e}-\check{\theta}_{m}, \check{\theta}_{m} \mapsto \check{\theta}_{m}$.
} 
this disc. By deforming $T_{s, \lambda}$ continuously to $T_{0, \lambda}$ and setting

$$
z_{\beta}\left(T_{s, \lambda}, \nabla\right)=\exp \left(-\int_{\beta} \omega(\zeta)\right) \operatorname{hol}_{\nabla}(\partial \beta),
$$

we get a globally defined holomorphic function $z_{\beta}$ on $\check{M}(\zeta)$ which is nothing but the coordinate $w$ given above. For $\lambda<0$, the holomorphic disc bounded by $T_{0, \lambda}$ has area $-\lambda$, and the corresponding holomorphic coordinate on the mirror is $z_{-\beta}=z_{\beta}^{-1}=w^{-1}$.

We can now construct the instanton-corrected mirror of $M(\zeta)=(M, \omega(\zeta), \Omega(\zeta))$, following the approach of Auroux [1], [2]. By the above proposition, we know that wall-crossing occurs at the wall $\{b \in B: \operatorname{Re}(\bar{\zeta} b)=0\}$. We remark that if we use the complex affine coordinates on $B$, then the wall is the straight line in $B$ invariant under monodromy. Now, the wall divides $B$ into two chambers: $B_{1}$ and $B_{2}$, as shown in Figure 1.

\section{Figure 1}

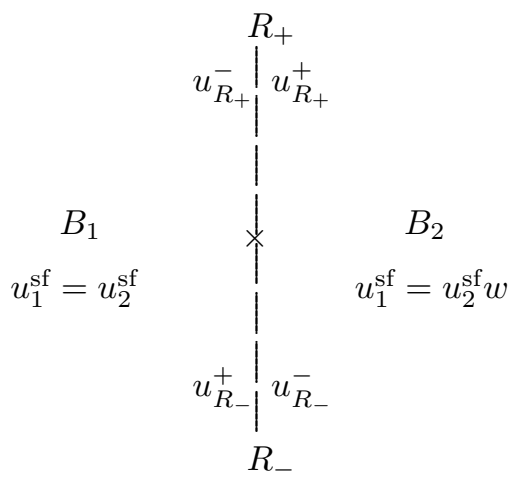

On $B \backslash\{\underline{b} \in B: \operatorname{Re}(\bar{\zeta} b)=0$ and $\operatorname{Im}(\bar{\zeta} b) \geq 0\}$ and $B \backslash\{b \in B: \operatorname{Re}(\bar{\zeta} b)=$ 0 and $\operatorname{Im}(\bar{\zeta} b) \leq 0\}$, we choose different branches of $\log$, say $\log _{1}$ and $\log _{2}$, so that $\log _{1}=\log _{2}$ on $B_{1}$ and $\log _{1}=\log _{2}+2 \pi i$ on $B_{2}$. Denote by

$$
\phi_{e}^{k}(\zeta)=-\frac{1}{\epsilon} \operatorname{Re}\left[\bar{\zeta}\left(b \log _{k} \frac{b}{r}-b\right)\right], u_{k}^{\mathrm{sf}}=\exp \left(-\phi_{e}^{k}(\zeta)-i \check{\theta}_{e}\right)
$$

the coordinates corresponding to the branch $\log _{k}$, for $k=1,2$. Hence the gluing of the complex charts of $\check{M}(\zeta)$ defined by the two sets of coordinates $\left(w, u_{1}^{\mathrm{sf}}\right)$ and $\left(w, u_{2}^{\mathrm{sf}}\right)$ are given by

$$
\begin{cases}u_{1}^{\mathrm{sf}}=u_{2}^{\mathrm{sf}} & \text { on } B_{1}, \\ u_{1}^{\mathrm{sf}}=u_{2}^{\mathrm{sf}} w & \text { on } B_{2},\end{cases}
$$

and this clearly does not define a global holomorphic coordinate.

What we need to do is to modify the gluing across the wall $\operatorname{Re}(\bar{\zeta} b)=0$ by disc instanton corrections as follows. Consider the rays

$$
\begin{aligned}
& R_{+}=\{b \in B: \operatorname{Re}(\bar{\zeta} b)=0 \text { and } \operatorname{Im}(\bar{\zeta} b)>0\}, \\
& R_{-}=\{b \in B: \operatorname{Re}(\bar{\zeta} b)=0 \text { and } \operatorname{Im}(\bar{\zeta} b)<0\} .
\end{aligned}
$$

Over the chamber $B_{1}$, let $u_{R_{+}}^{-}$be the coordinate $u_{1}^{\text {sf }}=u_{2}^{\text {sf }}$ as $b \in B$ approaches $R_{+}$in the clockwise direction, and $u_{R_{-}}^{+}$be the coordinate $u_{1}^{\mathrm{sf}}=u_{2}^{\mathrm{sf}}$ as $b \in B$ approaches $R_{-}$ 
in the counter-clockwise direction. Over the chamber $B_{2}$, let $u_{R_{+}}^{+}$be the coordinate $u_{2}^{\mathrm{sf}}$ as $b \in B$ approaches $R_{+}$in the counter-clockwise direction, and $u_{R_{-}}^{-}$be the coordinate $u_{1}^{\text {sf }}$ as $b \in B$ approaches $R_{-}$in the clockwise direction. (See Figure 1.) The corrected gluing should then be given by the following wall-crossing formulas.

$$
\begin{aligned}
& u_{R_{+}}^{-}=u_{R_{+}}^{+}(1+w), \\
& u_{R_{-}}^{+}=u_{R_{-}}^{-}\left(1+w^{-1}\right) .
\end{aligned}
$$

This defines a global holomorphic coordinate on $\check{M}(\zeta)$.

Now, we claim that the wall-crossing formulas (3.2), (3.3) can naturally be identified with the formulas (2.1), (2.2) which appear in the construction of Gaiotto, Moore and Neitzke. Indeed, by hyperkähler rotation, we know a priori that the mirror of the Calabi-Yau 2-fold $M(\zeta)=(M, \omega(\zeta), \Omega(\zeta))$ should be given by $\check{M}(\zeta)=M(-i \zeta)=$ $(M, \omega(-i \zeta), \Omega(-i \zeta))$. Also, observe that we have

$$
\begin{aligned}
\log \left|\chi_{e}(-i \zeta)\right| & =\frac{2 \pi}{\epsilon} \operatorname{Re}(\overline{-i \zeta} b)=-\frac{2 \pi}{\epsilon} \operatorname{Im}(\bar{\zeta} b)=\phi_{m}(\zeta) \\
\log \left|\chi_{m}^{\mathrm{sf}}(-i \zeta)\right| & =\frac{1}{\epsilon} \operatorname{Im}\left[\overline{-i \zeta}\left(b \log \frac{b}{r}-b\right)\right]=\frac{1}{\epsilon} \operatorname{Re}\left[\bar{\zeta}\left(b \log \frac{b}{r}-b\right)\right]=-\phi_{e}(\zeta) .
\end{aligned}
$$

Hence, the coordinates $w$ and $u^{\text {sf }}$ can naturally be identified with the semi-flat coordinates $\chi_{e}(-i \zeta)$ and $\chi_{m}^{\mathrm{sf}}(-i \zeta)$ respectively. (More precisely, this means that we have a canonical fiber-preserving diffeomorphism $M \rightarrow \check{M},\left(b_{1}, b_{2}, \theta_{e}, \theta_{m}\right) \mapsto\left(b_{1}, b_{2}, \check{\theta}_{m}=\right.$ $\left.\theta_{e}, \check{\theta}_{e}=-\theta_{m}\right)$ between $M \rightarrow B$ and $\check{M} \rightarrow B$ identifying the semi-flat local coordinates; see also Remark 3.3.)

So the two sets of equations (2.1), (2.2) and (3.2), (3.3) are both defining a global holomorphic coordinate on $M(-i \zeta)$ by correcting the semi-flat coordinate $u^{\mathrm{sf}}=\chi_{m}^{\mathrm{sf}}(-i \zeta)$, and the corrections involving $w=\chi_{e}(-i \zeta)$ are of the same form. The only difference is that the BPS rays $l_{+}, l_{-}$lie in the $\zeta$-plane, while $R_{+}, R_{-}$lie in $B$. However, we notice that the rays $R_{+}, R_{-}$can be rewritten as

$$
\begin{aligned}
& R_{+}=\left\{b \in B: b /(-i \zeta) \in \mathbb{R}_{<0}\right\} \\
& R_{-}=\left\{b \in B: b /(-i \zeta) \in \mathbb{R}_{>0}\right\}
\end{aligned}
$$

Now, when $b$ approaches $R_{+}$in the counter-clockwise direction, the BPS ray $l_{+}=$ $\left\{\zeta^{\prime}: b / \zeta^{\prime} \in \mathbb{R}_{<0}\right\}$ is rotating in the $\zeta$-plane in the counter-clockwise direction and approaching the fixed $-i \zeta$. Equivalently, $-i \zeta$ is approaching $l_{+}$in the clockwise direction. Likewise, when $b$ is approaching $R_{+}$in the clockwise direction, $-i \zeta$ is approaching $l_{+}$in the counter-clockwise direction; and similarly for $l_{-}$and $R_{-}$. We therefore come to the main conclusion of this note:

Suppose that $|\zeta|=1$. Then the equations (2.1), (2.2), with $\zeta$ replaced by $-i \zeta$, which describe the discontinuity of the holomorphic coordinate $\chi_{m}(-i \zeta)$ across the BPS rays $l_{ \pm}$, are equivalent to the wall-crossing formulas (3.2), (3.3) which appear in the construction of the instanton-corrected mirror of $M(\zeta)=(M, \omega(\zeta), \Omega(\zeta))$. 
In particular, we now see clearly how disc instanton corrections (given by nontrivial holomorphic discs with boundary on special Lagrangian torus fibers) contribute to the construction of the Ooguri-Vafa metric.

We end this note by a couple of remarks.

Remark 3.2. As we mentioned in the introduction, in the case of the Ooguri-Vafa metric, the Kontsevich-Soibelman wall-crossing formula of first kind is trivial. This is because the wall of first kind is empty and thus the numerical Donaldson-Thomas invariants, which are given by an integer-valued function $\Omega: \Gamma \rightarrow \mathbb{Z}$, is constant. ${ }^{10}$ More precisely, we have, for all $b \in B, \Omega\left(\gamma_{e}\right)=\Omega\left(-\gamma_{e}\right)=1$ and $\Omega(\gamma)=0$ for any $\gamma \notin\left\{ \pm \gamma_{e}\right\}$. In turn, this should be interpreted as the fact that only $\pm \gamma_{e}$, now regarded as elements in $H_{1}\left(\check{M}(\zeta)_{b}, \mathbb{Z}\right)$, bounds nontrivial holomorphic discs in $\check{M}(\zeta)$ with boundary on the dual special Lagrangian torus fibers. This is closely related to the comment stated in 1.5(2) on p.16 in [15], where Kontsevich-Soibelman speculated that the numerical Donaldson-Thomas invariants $\Omega(\gamma)$ should be counting certain holomorphic discs in $\check{M}(\zeta)$ "near infinity". As pointed out to me by Yan Soibelman, one interesting question is to interpret the wall-crossing formulas in terms of $3 d$ CalabiYau categories.

Remark 3.3. [The Ooguri-Vafa metric and SYZ mirror transformations] In [6], Gaiotto-Moore-Neitzke decomposed the positive harmonic function $V=V\left(b_{1}, b_{2}, b_{3}\right)$ and the 1-form $A=A\left(b_{1}, b_{2}, b_{3}\right)$ into a sum of semi-flat part and instanton part (see also [16]). More precisely, we can write

$$
\begin{aligned}
& V=V^{s f}+V^{i n s t} \\
& A=A^{s f}+A^{i n s t}
\end{aligned}
$$

where

$$
\begin{aligned}
V^{s f} & =-\frac{1}{4 \pi \epsilon}\left(\log \frac{b}{r}+\log \frac{\bar{b}}{r}\right) \\
V^{i n s t} & =\frac{1}{2 \pi \epsilon} \sum_{n \neq 0} K_{0}\left(\frac{2 \pi}{\epsilon}|n b|\right) e^{i n \theta_{e}}, \\
A^{s f} & =\frac{i}{8 \pi^{2}}\left(\log \frac{b}{r}-\log \frac{\bar{b}}{r}\right) d \theta_{e} \\
A^{i n s t} & =-\frac{1}{4 \pi \epsilon}\left(\frac{d b}{b}-\frac{d \bar{b}}{\bar{b}}\right) \sum_{n \neq 0}(\operatorname{sgn} n)|b| K_{1}\left(\frac{2 \pi}{\epsilon}|n b|\right) e^{i n \theta_{e}},
\end{aligned}
$$

and $K_{0}, K_{1}$ are modified Bessel functions. Accordingly, we can decompose the holomorphic two form $\Omega(\zeta)$ and the symplectic form $\omega(\zeta)$ into a sum of semi-flat and instanton parts.

$$
\begin{aligned}
& \Omega(\zeta)=\Omega^{s f}(\zeta)+\Omega^{i n s t}(\zeta) \\
& \omega(\zeta)=\omega^{s f}(\zeta)+\omega^{i n s t}(\zeta)
\end{aligned}
$$

\footnotetext{
${ }^{10}$ Caution: Do not confuse the $\Omega$ here with the holomorphic two-form $\Omega(\zeta)$.
} 
It is straightforward to show that we have

$$
\Omega^{s f}(\zeta)=\frac{d \chi_{e}^{s f}(\zeta)}{\chi_{e}^{s f}(\zeta)} \wedge \frac{d \chi_{m}^{s f}(\zeta)}{\chi_{m}^{s f}(\zeta)},
$$

which agrees with the formula in Section 2.

In the case $|\zeta|=1$, we compute the semi-flat parts $\Omega^{s f}(\zeta)$ and $\omega^{s f}(\zeta)$, and they are respectively given by

$$
\begin{aligned}
\Omega^{s f}(\zeta) & =\left(\frac{2 \pi}{\epsilon} d \operatorname{Re}(\bar{\zeta} b)+i d \theta_{e}\right) \wedge\left(\frac{2 \pi}{\epsilon} d \operatorname{Re}\left(\bar{\zeta} Z_{m}\right)+i d \theta_{m}\right), \\
\omega^{s f}(\zeta) & =-\frac{2 \pi}{\epsilon} d \operatorname{Im}(\bar{\zeta} b) \wedge d \theta_{m}+\frac{2 \pi}{\epsilon} d \operatorname{Im}\left(\bar{\zeta} Z_{m}\right) \wedge d \theta_{e},
\end{aligned}
$$

Now, if we set $\check{\theta}_{e}=-\theta_{m}, \check{\theta}_{m}=\theta_{e}$ (they are the dual fiber coordinates on $\check{M}=M$ ), then

$$
\begin{aligned}
\omega^{s f}(-i \zeta) & =\frac{2 \pi}{\epsilon} d \operatorname{Im}(i \bar{\zeta} b) \wedge d\left(-\theta_{m}\right)+\frac{2 \pi}{\epsilon} d \operatorname{Im}\left(i \bar{\zeta} Z_{m}\right) \wedge d \theta_{e} \\
& =\frac{2 \pi}{\epsilon} d \operatorname{Re}(\bar{\zeta} b) \wedge d \check{\theta}_{e}+\frac{2 \pi}{\epsilon} d \operatorname{Re}\left(\bar{\zeta} Z_{m}\right) \wedge d \check{\theta}_{m}
\end{aligned}
$$

We can then show that

$$
\begin{aligned}
\mathcal{F}^{s f}\left(e^{i \omega^{s f}}(-i \zeta)\right) & =\Omega^{s f}(\zeta), \\
\left(\mathcal{F}^{s f}\right)^{-1}\left(\Omega^{s f}(\zeta)\right) & =e^{i \omega^{s f}(-i \zeta)},
\end{aligned}
$$

where $\mathcal{F}^{s f}$ is the semi-flat SYZ mirror transformation introduced in Chan-Leung [4], [5]. Moreover, since $V^{\text {inst }}$ and $A^{\text {inst }}$ are periodic in $\theta_{e}$ and have no constant terms in their Fourier series expansions, we have

$$
\left(\mathcal{F}^{s f}\right)^{-1}\left(\Omega^{i n s t}(\zeta)\right)=0 .
$$

It is thus very natural to ask whether one can construct an SYZ mirror transformation $\mathcal{F}$ such that

$$
\begin{aligned}
\mathcal{F}\left(e^{i \omega(-i \zeta)}\right) & =\Omega(\zeta) \\
(\mathcal{F})^{-1}(\Omega(\zeta)) & =e^{i \omega(-i \zeta)}
\end{aligned}
$$

This is related to the question of writing down the Kähler structure on the mirror manifold in terms of the holomorphic volume form on the original manifold. We hope to return to this in a later paper.

\section{Acknowledgements}

I am very grateful to Prof. Shing-Tung Yau for suggesting this problem, to Andy Neitzke for carefully explaining their constructions, and to Prof. Yan Soibelman for answering my questions through emails and pointing out many inaccuracies in earlier versions of this article. I would also like to thank Prof. Denis Auroux, Prof. Mark Gross, Prof. Naichung Conan Leung and Prof. Eric Zaslow for numerous useful discussions. Finally, I thank the referees for several helpful comments. This research was supported by Harvard University and the Croucher Foundation Fellowship. 


\section{References}

[1] D. Auroux, Mirror symmetry and T-duality in the complement of an anticanonical divisor. J. Gokova Geom. Topol. GGT, 1 (2007), 51-91. arXiv:0706.3207.

[2] _ S Special Lagrangian fibrations, wall-crossing, and mirror symmetry. Surveys in differential geometry. Vol. 13, 1-47, Int. Press, Somerville, MA, 2009. arXiv:0902.1595.

[3] R. Castaño Bernard and D. Matessi, Lagrangian 3-torus fibrations. J. Differential Geom. 81 (2009), no. 3, 483-573. math.SG/0611139.

[4] K.-W. Chan and N.-C. Leung, Mirror symmetry for toric Fano manifolds via SYZ transformations. Adv. Math. 223 (2010), no. 3, 797-839. arXiv:0801.2830.

[5] _ On SYZ mirror transformations. To appear in Advanced Studies in Pure Mathematics, "New developments in Algebraic Geometry, Integrable Systems and Mirror Symmetry". arXiv:0808.1551.

[6] D. Gaiotto, G. Moore and A. Neitzke, Four-dimensional wall-crossing via three-dimensional field theory. Preprint 2008. arXiv:0807.4723.

[7] M. Gross, Examples of special Lagrangian fibrations. Symplectic geometry and mirror symmetry (Seoul, 2000), 81-109, World Sci. Publ., River Edge, NJ, 2001. math.AG/0012002.

[8] M. Gross and B. Siebert, From real affine geometry to complex geometry. Preprint 2007. math.AG/0703822.

[9] M. Gross and P. M. H. Wilson, Large complex structure limits of K3 surfaces. J. Differential Geom. 55 (2000), no. 3, 475-546. math.DG/0008018.

[10] N. Hitchin, Hyper-Kähler manifolds. Séminaire Bourbaki, Vol. 1991/92. Astérisque No. 206 (1992), Exp. No. 748, 3, 137-166.

[11] _ The moduli space of special Lagrangian submanifolds. Ann. Scuola Norm. Sup. Pisa Cl. Sci. (4) 25 (1997), no. 3-4, 503-515. dg-ga/9711002.

[12] N. Hitchin, A. Karlhede, U. Lindström and M. Roček, Hyper-Kähler metrics and supersymmetry. Comm. Math. Phys. 108 (1987), no. 4, 535-589.

[13] M. Kontsevich and Y. Soibelman, Homological mirror symmetry and torus fibrations. Symplectic geometry and mirror symmetry (Seoul, 2000), 203-263, World Sci. Publ., River Edge, NJ, 2001. math.SG/0011041.

[14] _ Affine structures and non-Archimedean analytic spaces. The unity of mathematics, 321-385, Progr. Math., 244, Birkhauser Boston, Boston, MA, 2006. math.AG/0406564.

[15]_, Stability structures, motivic Donaldson-Thomas invariants and cluster transformations. Preprint 2008. arXiv:0811.2435.

[16] H. Ooguri and C. Vafa, Summing up Dirichlet instantons. Phys. Rev. Lett. 77 (1996), no. 16, 3296-3298. hep-th/9608079.

[17] A. Strominger, S.-T. Yau and E. Zaslow, Mirror symmetry is T-duality. Nuclear Phys. B, 479 (1996), no. 1-2, 243-259. hep-th/9606040.

Department of Mathematics, Harvard University, Cambridge, MA 02138

E-mail address: kwchan@math.harvard.edu 